\section{PERSPECTIVAS \\ EN INTELIGENCIA \\ -2020}

\title{
Implementación de un sistema de geoposicionamiento con transmisión de coordenadas por radiofrecuencia ${ }^{75}$
}

\author{
Implementation of a geopositioning system with radio frequency \\ transmission of coordinates
}

\section{Álvaro Andrés Guzmán Castañeda ${ }^{1}$, Juan Wilfredo Pinto Uribe ${ }^{2}$ y Diego Arley Velosa Castañeda ${ }^{3^{*}}$}

(1) Universidad Piloto de Colombia, Bogotá - Colombia, aguzmanc@imi.mil.co

(2) Universidad Industrial de Santander, Bucaramanga - Colombia, jpintou@imi.mil.co

(3) Universidad Distrital Francisco José de Caldas, Bogotá - Colombia, diegovelosa@gmail.com

* Autor a quien se dirige la correspondencia

\section{Resumen}

La implementación de sistemas de geolocalización ha tenido gran auge en la última década, bajola primicia depodercontrolaryproporcionarseguridad en eldesplazamiento de vehículos o personas en el desarrollo de diferentes actividades. No obstante, los diferentes dispositivos dispuestos para dichas funciones han sido parcialmente comercializados para interactuar en campo con el uso de redes de comunicaciones móviles o, en su defecto, dispositivos de posicionamiento global independientes, con comunicación satelital para la visualización en plataformas digitales. Estos sistemas generan ciertas limitantes, tanto en tamaño como en consumo de energía, debido a los dispositivos dispuestos para uso en aplicaciones generales. Es por esto que se realizó un estudio tecnológico de los diferentes dispositivos de geoposicionamiento, teniendo en cuenta tamaño, consumo energético y envío de información por radiofrecuencia. De esta forma se diseñó e implementó un sistema con un nodo remoto para uso en inteligencia estratégica militar, para desplazamientos en áreas sin coberturas de redes de comunicaciones móviles. Y una estación base encargada de la recepción de información y de envío a una plataforma de visualización satelital. Como resultado, se obtuvo un módulo remoto de bajas dimensiones que permanece en estado de reposo para ahorrar energía y solo se activa cuando recibe un mando desde el módulo base, teniendo un alcance de enlace superior a veinte $\mathrm{kms}$, siempre y cuando se asegure línea de vista.

La ventaja de tener un dispositivo programable en el nodo remoto es el poder controlar variables que no se logran manejar en otros dispositivos similares, tales como: ahorro de energía, tiempos de trasmisión de datos y codificación propia para seguridad de la transmisión, convirtiéndose así en un sistema más confiable para uso en operaciones militares.

Palabras clave: Geolocalización; Radiofrecuencia; Inteligencia Estratégica.

75 Artículo de investigación del Batallón de Investigación Desarrollo e Innovación en Inteligencia 


\section{Abstract}

The implementation of geolocation systems has had a great boom in the last decade, under the scoop of being able to control and provide security in the movement of vehicles or people in the development of different activities. However, the different devices arranged for said functions have been partially commercial to interact in the field with the use of mobile communication networks or, failing that, independent global positioning devices, with satellite communication for viewing on digital platforms. These systems have certain limitations both in size and in power; consumption due to the devices arranged for use in general applications. This is why a technological study of the different geopositioning devices was carried out, taking into account the size, energy and sending of information by radio frequency. In this way, a system with a remote node was designed and implemented for use in military strategic intelligence, for movements in areas without mobile communication network coverage. In addition, a base station in charge of receiving information and sending it to a satellite viewing platform. As a result, a remote module of small dimensions was obtained that remains in a standby state to save energy and is only activated when it receives a command from the base module, having a link range greater than twenty kms as long as line of sight is assured.

The advantage of having a programmable device in the remote node is being able to control variables that cannot be handled in other similar devices, such as energy saving, data transmission times and own coding for transmission security, thus becoming a more reliable system for use in military operations.

Keywords: Geolocation; Radiofrequency; IoT; Strategic Intelligence.

\section{Introducción}

En el desarrollo de las tecnologías relacionadas con la Industria 4.0, muchas aplicaciones han tenido una evolución tendiente a dar soluciones a los problemas más comunes en las comunicaciones, como el alcance, la cobertura, transmisión de datos, alimentación eléctrica, seguridad y conectividad de sensores, los cuales permitan acelerar la productividad y conectividad de las fábricas y articular la producción con el usuario final. En este sentido, el desarrollo de las comunicaciones móviles tiene grandes implicaciones, sobre las cuales se ha venido trabajando principalmente en el Internet de las Cosas (IoT) y tecnologías celulares de quinta generación (5G) (Corzo \& Álvarez-Aros, 2020).

Esta necesidad no ha sido ajena a la utilidad en el ámbito militar, una de las tecnologías que ha sido más utilizadas es el IoT, el cual se utiliza en muchas aplicaciones, principalmente de coordinación logística, como el seguimiento de los signos vitales, seguridad biológica y salud para los soldados, suministro de insumos, alimentación, municiones $y$, en general, el material de combate, así como la comunicación entre dispositivos y aplicaciones para la atención y prevención de desastres (Wang et al., 2019). Además, la información sobre la posición de elementos de interés es fundamental en el contexto militar y generalmente se basa en la capacidad de integrar tecnologías que deben contar con un alto nivel de seguridad, el cual se puede acoplar a la tecnología IoT (Gotarane \& Raskar, 2019). 
El problema más común en las aplicaciones militares, es la ubicación de dispositivos y sensores a través de posicionamiento satelital, ya que muchas tecnologías no funcionan de manera adecuada en condiciones que los entornos militares requieren, debido a la limitada cobertura causada por la topografía e interferencia con otros sistemas de comunicación. En algunas situaciones, la implementación de estas aplicaciones se hace sobre plataformas virtuales que no cuentan con la seguridad adecuada para el tráfico de información de tipo confidencial y, teniendo en cuenta que se hace uso del ciberespacio como canal de comunicación, el control sobre los datos manejados se vuelve vulnerable a cualquier tipo de ataque o interceptación tanto de empresas privadas como organismos del Estado ajenos al trabajo ejercido por las Fuerzas Militares (Chaves \& Jurado, 2019).

Otro factor que influye es el alto consumo energético asociado a la obtención de la posición de un dispositivo electrónico al tener que conectarse continuamente con el sistema satelital, por lo que utilizar un solo mecanismo de ahorro de energía no garantiza que se pueda alargar lo suficiente su funcionamiento, luego se busca combinar diferentes estrategias que incluyen, por ejemplo, el uso de antenas reconfigurables como las estudiadas por Di Renzo (2017).

En esta investigación se propone la implementación de un sistema integrado de sensores con geolocalización satelital. Para lograrlo, se hace en primer lugar un estudio de tecnologías relacionadas, identificando sus principales características y analizando sus deficiencias, con el fin de desarrollar un prototipo funcional. En la siguiente sección se muestra la metodología para la solución propuesta con las características funcionales, se presenta el desarrollo del dispositivo hasta llegar al producto final. Finalmente se describen los resultados obtenidos, realizando un análisis de las limitaciones y alcances del producto final y discutiendo la forma de cómo se debe realizar una implementación a mayor escala.

\section{Sistemas de Geolocalización}

En el campo de seguimiento de objetivos y control de desplazamientos en tiempo real, el envío y recolección de coordenadas es la base fundamental para poder generar bases de datos de ubicaciones, análisis de comportamientos o simplemente frecuencias de permanencia en lugares específicos por los diferentes individuos que sean el objetivo de investigación.

Los datos de geolocalización tienen una variedad de usos, cada uno de los cuales se puede personalizar para determinadas aplicaciones o entornos. Actualmente estos usos incluyen la ubicación y/o personalización de los contenidos distribuidos, la aplicación de restricciones de acceso y distribución, de acuerdo con la ubicación geográfica, prevención de fraudes y análisis de tráfico de redes. En muchos de los casos, la recolección de los datos proporcionados por los equipos de geolocalización se ejecuta por medio del enlace con redes de comunicaciones comerciales disponibles en las áreas de aplicación o simplemente con equipos ajustados para visualización directa de coordenadas, lo que los hace limitarse en tamaños y consumo energético para el funcionamiento. Teniendo en cuenta que en el mercado existe una gama amplia de geolocalizadores o dispositivos de geoposicionamiento, el enfoque será en los que poseen muy bajas dimensiones y que brindan la capacidad de seguimiento de objetivos en tiempo real. 


\section{Características de los dispositivos de seguimiento remoto más habituales}

Algunos de los dispositivos más comunes y comerciales usados para control y ubicación de individuos u objetivos se describen en la Tabla 1, en donde se realiza un comparativo respecto a sus características.

-TABLA 1. Comparación de tecnologías dispositivos de seguimiento remoto

\begin{tabular}{|c|c|c|c|c|c|}
\hline & $\begin{array}{l}\text { Módulos GPS } \\
\text { Satelitales }\end{array}$ & $\begin{array}{l}\text { Módulos } \\
\text { GPS-GSM }\end{array}$ & Radiotelemetría & $\begin{array}{l}\text { Módulos } \\
\text { GPS-RF }\end{array}$ & Minitrackers \\
\hline $\begin{array}{l}\text { Tipo de } \\
\text { conectividad }\end{array}$ & Satelital & $\begin{array}{l}\text { Red móvil } \\
\text { celular }\end{array}$ & Radiofrecuencia & $\begin{array}{l}\text { Radiofre- } \\
\text { cuencia }\end{array}$ & Bluetooth \\
\hline Tamaño & Alto & Medio alto & Medio alto & Medio & Bajo \\
\hline $\begin{array}{l}\text { Consumo } \\
\text { energético }\end{array}$ & Alto & Medio alto & Medio & Medio alto & Bajo \\
\hline Alcance & Alto & Medio & Medio alto & Medio alto & Muy Bajo \\
\hline Cobertura & Alta & Alta & Baja & Baja & Muy Baja \\
\hline $\begin{array}{l}\text { Sensibilidad } \\
\text { de } \\
\text { interferencia }\end{array}$ & Alta & Baja & Baja & Medio Bajo & Baja \\
\hline $\begin{array}{l}\text { Requiere pago } \\
\text { de servicio }\end{array}$ & Sí & Sí & No & No & No \\
\hline $\begin{array}{l}\text { Costos USD } \\
\text { (Aprox) }\end{array}$ & $\$ 464$ & $\$ 620$ & $\$ 360$ & $\$ 130$ & $\$ 40$ \\
\hline Ventajas & $\begin{array}{c}\text { Buena cobertura } \\
\text { a nivel mundial }\end{array}$ & $\begin{array}{c}\text { Buena cobertura } \\
\text { a nivel urbano }\end{array}$ & $\begin{array}{c}\text { Bajo nivel de } \\
\text { interferencia por } \\
\text { propagación }\end{array}$ & $\begin{array}{l}\text { Bajo consumo, } \\
\text { largo alcance }\end{array}$ & $\begin{array}{c}\text { Bajo consumo } \\
\text { y tamaño } \\
\text { reducido }\end{array}$ \\
\hline Desventajas & $\begin{array}{l}\text { Un alto consumo } \\
\text { y tamaño para } \\
\text { su uso en ciertas } \\
\text { aplicaciones }\end{array}$ & $\begin{array}{l}\text { Solo funciona } \\
\text { bajo cobertura } \\
\text { de redes GSM }\end{array}$ & $\begin{array}{c}\text { Bajo nivel de } \\
\text { exactitud para } \\
\text { ubicación y baja } \\
\text { cobertura }\end{array}$ & $\begin{array}{c}\text { Presenta } \\
\text { pérdidas por } \\
\text { propaga-ción e } \\
\text { interferen-cias } \\
\text { y una baja } \\
\text { cobertura }\end{array}$ & $\begin{array}{l}\text { Presenta un } \\
\text { alcance muy } \\
\text { limitado }\end{array}$ \\
\hline
\end{tabular}

Fuente: elaboración propia

En el campo de dispositivos de seguimiento por geoposicionamiento se encuentran bastantes sistemas muy bien posicionados. Algunos de ellos se enfocan en el monitoreo de vida silvestre, en especial de aves, y otros se desarrollan para el control de vehículos o desplazamientos de personas con limitaciones físicas. Algunos de ellos se relacionan a continuación: 
Técnicas de marcaje de aves Marinas para el seguimiento remoto: En este tipo de proyectos se usan dispositivos de muy bajas dimensiones, ya que muchos de ellos deben contar con el menor peso y tamaño posible para no generar lesiones o problemas de desarrollo del ejemplar. Se destacan los emisores de radio, que emiten en una frecuencia de radio determinada que identifica a cada individuo, produciendo una serie de pulsos por minuto (por ejemplo 45 pulsos/min) de una duración determinada (por ejemplo 20 $\mathrm{ms} /$ pulso). El investigador con un receptor de radio mediante una antena direccional puede recibir la señal del emisor con mayor o menor intensidad, según la distancia a la que se encuentre y el estado de la batería. Para conocer la ubicación aproximada del individuo basta con una localización, pero para conocer con cierta exactitud la posición se necesita triangular tomando al menos dos direcciones, simultáneas o con equipo estático, de la señal con la antena direccional y determinando la posición en el punto o zona de corte de las direcciones tomadas desde dos puntos diferentes (Bécares et al., 2010). Esto lo hace vulnerable a fallos por precisión.

También se usan los geolocalizadores (GLS) que proporcionan un registro casi continuo de la luz ambiental a lo largo de todos los días de un año, almacenando la lectura máxima de luz por períodos de, por ejemplo, 10 minutos. Pese a todo, su poca precisión (error de $100-150 \mathrm{~km}$ ) hace de este sistema un método inapropiado para definir o conocer áreas precisas (Bécares et al., 2010).

El registrador de compás es otro sistema que almacena en su memoria las direcciones de desplazamiento a través de dos brújulas (una mide las diferencias hacia el norte y la otra hacia el este) incrementando así su precisión. En estos aparatos el factor limitante es la memoria, no la batería, por lo que hay que programar intentando ajustar la máxima obtención de datos (más registros implican mayor exactitud en el desplazamiento) con la duración de la batería hasta el final del viaje. Este dispositivo es poco utilizado, ya que se trata de aparatos pesados, de más de $17 \mathrm{~g}$, con una duración de la batería relativamente corta, que hay que recuperar para la descarga de datos (Bécares et al., 2010).

Sistemas de control de posición angular aplicado a dispositivos $R \mathbf{f}$ : es un proyecto de investigación de tres ingenieros en Control, de la Universidad Distrital Francisco José de Caldas, que describe la modelación e implementación de un controlador difuso para enlazar unidades móviles UBA (Unidades Básicas de Atención) con centrales principales de salud. Para ello, utilizan un sistema de posicionamiento global (GPS), una base de datos de coordenadas de las centrales dispuestas en el área de prueba, un dispositivo (brújula electrónica) para la orientación del suscriptor, y un rotor cuyo propósito es lograr un posicionamiento inicial que genera el enlace con la unidad central. El producto propuesto hace que el GPS lea la ubicación, compare la posición cardinal a través de la brújula electrónica y relacione la ubicación con la base de datos de las antenas disponibles para que, finalmente, el rotor se direccione en la posición de conexión. (Olivera et al, 2011). En este sistema dependen de tener una base de datos, la cual sirve de referencia para comparar y determinar de dónde provienen las señales, lo que lo limita para un uso de seguimiento de un objetivo que no se encuentre siempre en un área específica y con la misma intensidad en la señal para generar el enlace estable, además de hacer uso de un computador para realizar la ejecución del controlador. 
Sistema de posicionamiento en interiores, utilizando señales de radio, estaciones FM comerciales y Deep Learning: es un sistema propuesto por el Grupo de Automática, Electrónica y Ciencias Computacionales del Instituto Tecnológico Metropolitano, Medellín, Colombia, para determinar el posicionamiento de individuos en interiores haciendo uso de estaciones de Radio FM y algoritmos Deep Learning. (Roman, J. et $\mathrm{al}, 2020$ ). Esto tiene una ventaja frente a otros sistemas, y es que evita los costos de implementación en infraestructura de antenas o equipos transceiver de Rf, usando las redes FM comerciales, pero al mismo tiempo lo hace débil frente al objetivo que se busca en la implementación del sistema de geoposicionamiento por radiofrecuencia, ya que en áreas sin cobertura de señales de FM cualquier enlace se perdería y, por ende, no se podría implementar un protocolo de entrenamiento para el software diseñado.

Orientación de pasajeros con discapacidad visual dentro del Sistema de Transporte Masivo Transmilenio, mediante Geolocalización Satelital: es un sistema diseñado por un grupo de investigación de la Universidad Distrital Francisco José de Caldas de Bogotá, Colombia, que busca mejorar el desplazamiento de pasajeros con discapacidad visual dentro del sistema de transporte Transmilenio. (Camargo, J et al, 2017). Se basa en la implementación de una interface de usuario con una base de datos y sistema de geolocalización que le permite al usuario tener alarmas auditivas para poder movilizarse. Tiene la gran ventaja de ser muy económico a la hora de su fabricación, por los componentes que lo conforman; sin embargo, para la funcionalidad del caso de operaciones militares se ve limitado por el tamaño y discreción que se necesitan en campo de ejecución, ya que se busca implementar un dispositivo muy fácil de mimetizar y que no genere ningún tipo de sospecha en el seguimiento de objetivos.

La tecnología de radiofrecuencia que está incursionando desde la industria 4.0 con gran éxito hacia las aplicaciones militares y con enfoque de interoperabilidad con dispositivos IoT son los equipos de tecnología LoRaWAN (Redes de baja potencia y área amplia), que brindan bajo consumo energético con la propiedad de trabajar en redes locales, regionales, nacionales y globales.

Adicionalmente, tienen la ventaja de ser dispositivos con los cuales se implementan estructuras de comunicación que no dependen de un operador comercial o de un tercero. Actualmente se está trabajando en equipos bajo esta tecnología que incluyen sistemas de geoposicionamiento embebido y con tamaños muy reducidos (Jalaian et al., 2018).

\section{Metodología}

El sistema propuesto para la geolocalización de objetivos por transmisión de radiofrecuencia tiene como fin desarrollar un dispositivo de cortas dimensiones, bajo consumo y peso reducido, para ser utilizado en áreas sin cobertura de redes móviles comerciales de comunicación. Este proyecto de transmisión de coordenadas se diseñó para estar compuesto por dos subsistemas. El subsistema de transmisión, o Nodo Remoto, que se encarga de realizar el fix de posicionamiento de GPS para luego transmitir los datos de coordenadas, y el subsistema de recepción, o Nodo central, que se encarga de recibir, procesar y enviar la coordenada del nodo remoto para visualizar los datos en una plataforma de monitoreo satelital. 
En la Figura 1 se detallan los bloques y subsistemas del proyecto en general:

-FIGURA 1• Diagrama de bloques sistema de geolocalización con módulos de radiofrecuencia

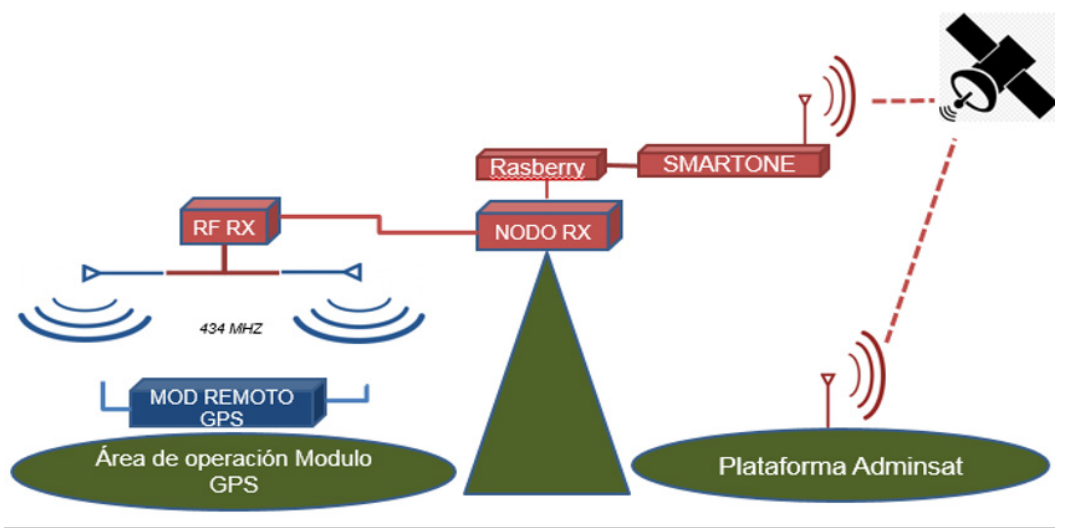

Fuente: elaboración propia

Subsistema de Transmisión o Nodo Remoto (NREM): El nodo remoto del proyecto ofrece funciones de radiogoniometría y ubicación geográfica. Esta información es obtenida de forma inalámbrica mediante un enlace de radiofrecuencia de largo alcance. En la figura 2 se pueden observar los módulos que componen al Nodo Remoto.

-FIGURA 2• Diagrama de Bloques del Nodo Remoto

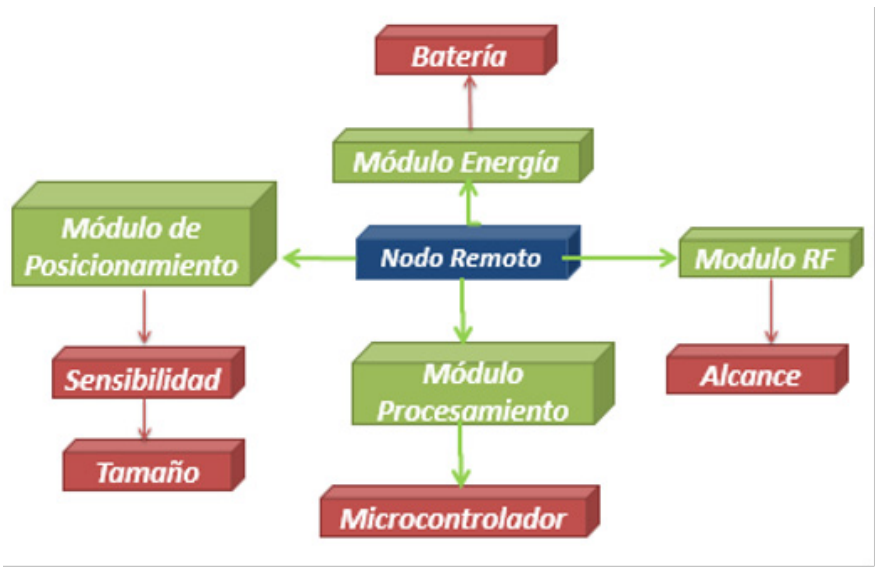

Fuente: elaboración propia 
- Módulo Energía: conformado por la batería, el regulador de voltaje del microcontrolador y los reguladores de voltaje tipo ON/OFF. Se encarga de entregar el voltaje de alimentación a los demás componentes y proveer control sobre el encendido y apagado de estos, con el fin de manejar el consumo energético del sistema.

- Módulo de posicionamiento: corresponde al GPS. Este módulo permite conocer la ubicación geográfica del dispositivo, tomando la coordenada y transfiriendo la información al módulo de procesamiento o microcontrolador. Se seleccionó un GPS que tuviera muy buena relación tamaño - sensibilidad, para poder mantener las condiciones de bajas dimensiones del nodo sin sacrificar recepción de coordenadas.

- Módulo Radiofrecuencia (RF): consiste en un transceptor de radiofrecuencia que permite la comunicación bidireccional tipo Half-Dúplex entre el nodo transmisor y el nodo receptor del proyecto a una frecuencia de $433 \mathrm{MHz}$.

- Módulo de procesamiento: se encuentra integrado por el microcontrolador y se encarga de la recepción de información proveniente del módulo RF, el procesamiento y ajuste de la coordenada recibida por el GPS y el envío de la respectiva trama de información con los parámetros necesarios para la correcta comunicación con el nodo de recepción.

- Diseño de Tarjeta: para poder optimizar las pruebas del sistema y reducir fallos por disposición de hardware, se llevó a cabo el diseño de la tarjeta para el Nodo Receptor en el software llamado Altium Designer, en el cual se desarrolló el diagrama del circuito, como se detalla en la Figura 3.

•FIGURA 3・Diseño Circuito Nodo Remoto

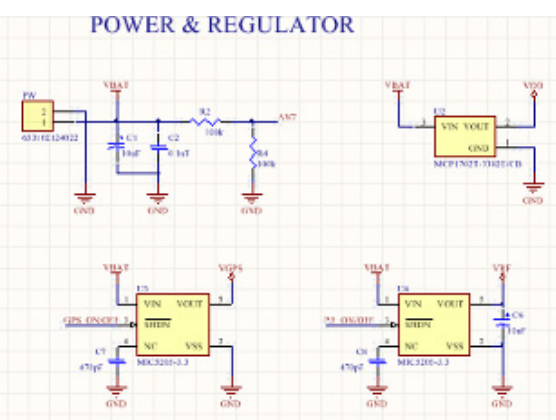

MICROCONTROLLER
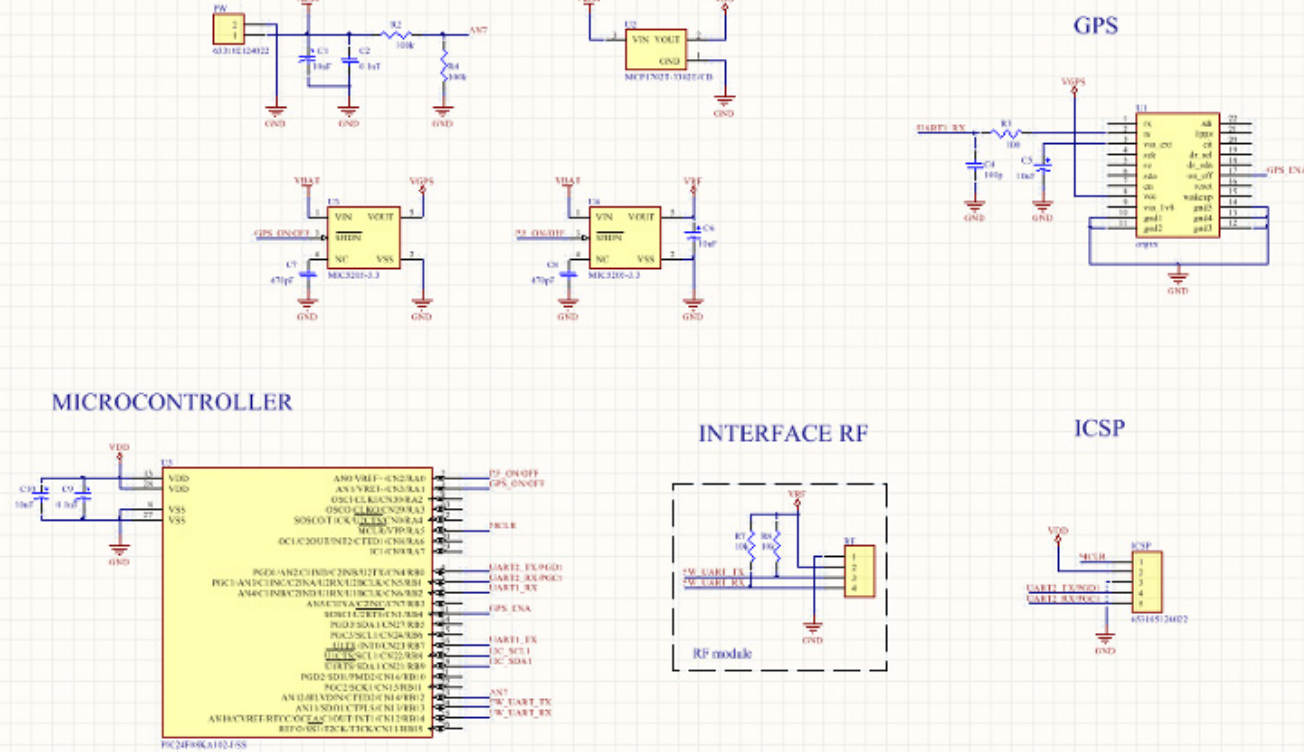

Fuente: elaboración propia 
Luego se procedió a diseñar en el mismo software la placa o PCB a dos capas para el ensamble de los dispositivos, distribuyendo en la máscara inferior el microcontrolador de procesamiento con su respectivo circuito de regulación de voltaje y conectores de periféricos, y en la máscara superior la huella del módulo GPS, como muestra la Figura 4, esto con el fin de obtener un tamaño bastante reducido para cumplir con las especificaciones técnicas requeridas para su operatividad.

•FIGURA 4• Diseño PCB nodo remoto
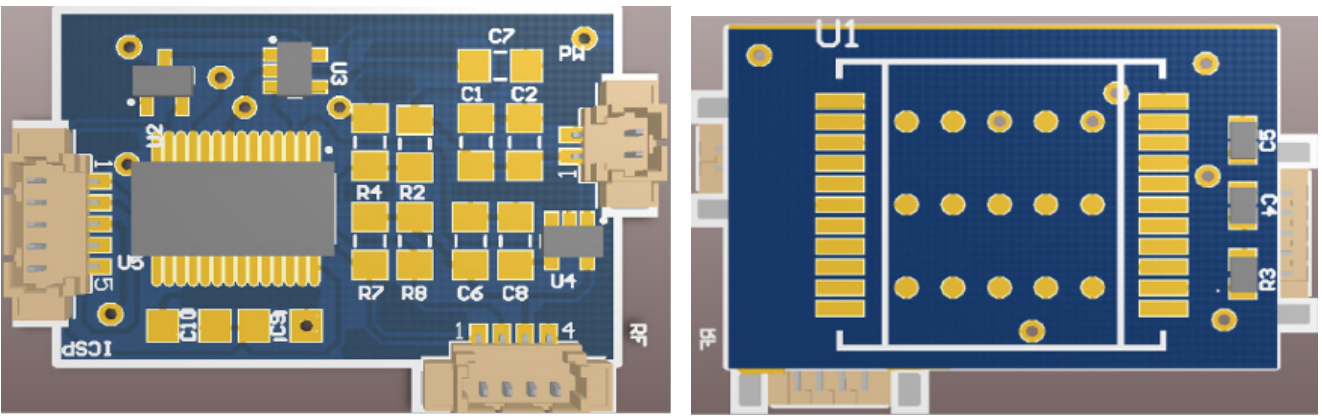

Fuente: elaboración propia

Subsistema de Recepción o Nodo Central: para ejecutar la comunicación con el nodo remoto, el nodo central se encarga de enviar una trama de activación y luego recibe la información con la coordenada recolectada en el área, todo a través de los módulos de radiofrecuencia. Luego procesa los datos y los acondiciona para ser enviados por un módulo satelital a la plataforma de visualización Adminsat. En la Figura 5 se observan los módulos que integran el subsistema de Recepción.

•FIGURA 5• Diagrama de bloques del nodo central

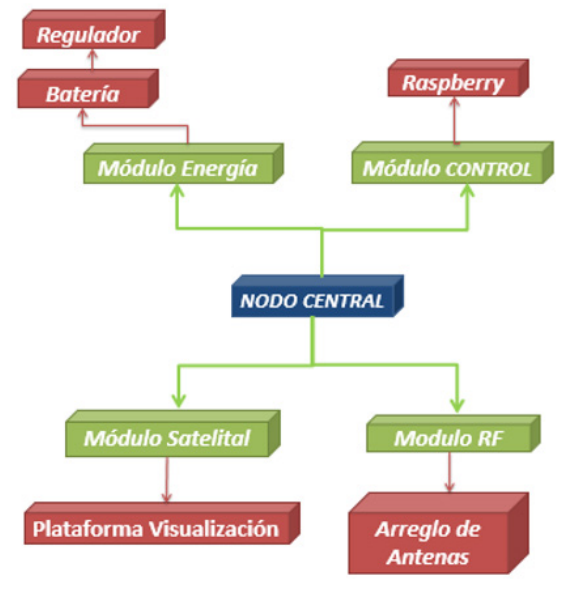

Fuente: elaboración propia 
- Módulo Energía: está conformado por una batería de Polímero de Litio de 14.8V, 5200 $\mathrm{mA}$ y un regulador de $5 \mathrm{v} 3 \mathrm{~A}$ que se encargan de alimentar a los módulos transceiver de radiofrecuencia, al módulo de procesamiento y al módulo satelital.

- Modulo RF: lo integran dos transceiver KYL200 de 500 milivatios y un arreglo de antenas omnidireccionales con una frecuencia de trabajo de 430-450 Mhz y ganancia de la antena de $8 \mathrm{dBi}$, configurados para recepción y transmisión del protocolo de comunicación ejecutado por el módulo de procesamiento.

- Módulo de Control: está conformado por una Raspberry Pi 3 que es la encargada de almacenar, procesar y controlar el envío y recepción de información para la ubicación del nodo remoto. A través de ella se interconectan los módulos transceiver, usados en la comunicación bidireccional, con el nodo remoto y el módulo satelital SmartOne, usado en el envío de la coordenada para la visualización en plataforma Adminsat. La ventaja de tener la información almacenada en este equipo es que se pueden establecer diferentes formas de procesamiento o de reenvío por sistemas de comunicación alternos, como radioenlaces, archivos de texto o almacenar la información en bases de datos.

- Módulo Satelital: se encarga de tomar la información procesada por el módulo de control y trasmitirla por satélite a una plataforma virtual. Esta función es ejecutada por el SmartOne, el cual es un dispositivo de conexión satelital de bajo consumo, ideal para el seguimiento de activos y monitoreo de eventos; posee un puerto serial para la comunicación con el módulo de procesamiento. La visualización de las coordenadas enviadas se grafica en una plataforma digital llamada Adminsat, a la cual se tiene acceso por pago del servicio prestado por un proveedor.

\section{Resultados}

Hardware Final: al término del proceso de ensamblado e implementación de los circuitos y elementos dispuestos para el funcionamiento del sistema de geoposicionamiento por transmisión de coordenadas, se logra obtener el dispositivo remoto con muy bajas dimensiones, cercanas al tamaño de una moneda, como se observa en las figuras 6 y 7, con el fin de facilitar el mimetizaje en equipos o accesorios para su transporte y respectivo uso.

•FIGURA 6• Nodo remoto vista superior

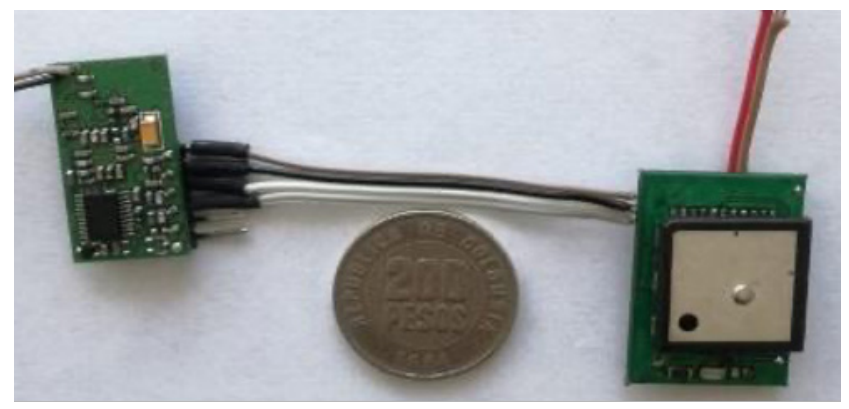

Fuente: elaboración propia 
-FIGURA 7• Nodo remoto vista inferior

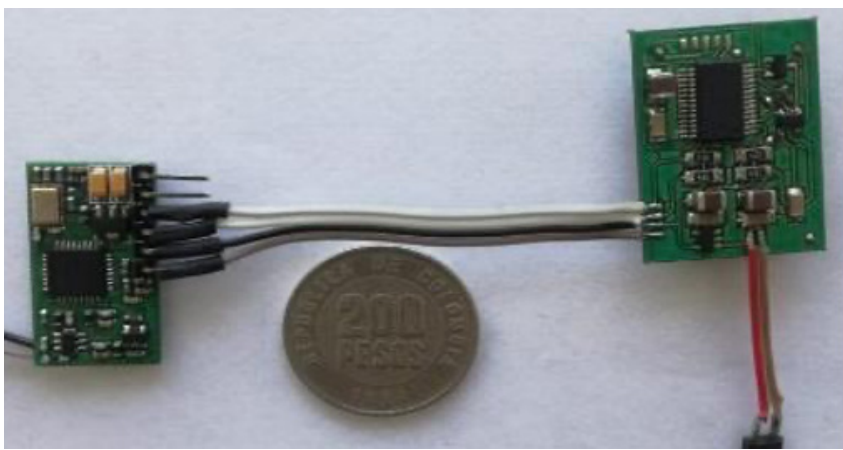

Fuente: elaboración propia

El módulo de recepción está diseñado para uso en una estación de control y monitoreo, por lo cual su tamaño no es relevante para la puesta en operación; debido a esto, la implementación de los elementos que lo conforman se ha distribuido en una caja de fácil manipulación y conexión en diferentes entornos. En la Figura 8 se pueden observar los equipos y dispositivos interconectados que hacen parte de este módulo, entre ellos se destacan la Raspberry Pi 3, los Transceiver de radiofrecuencia, una tarjeta multiplexora que se implementó para interconectar el puerto serie de la Raspberry con los puertos serie de lo módulos Rf y del módulo satelital, que también sobresale por ser de color blanco; y, por último, la batería que proporciona la energía a todo el sistema.

-FIGURA 8• Caja de recepción

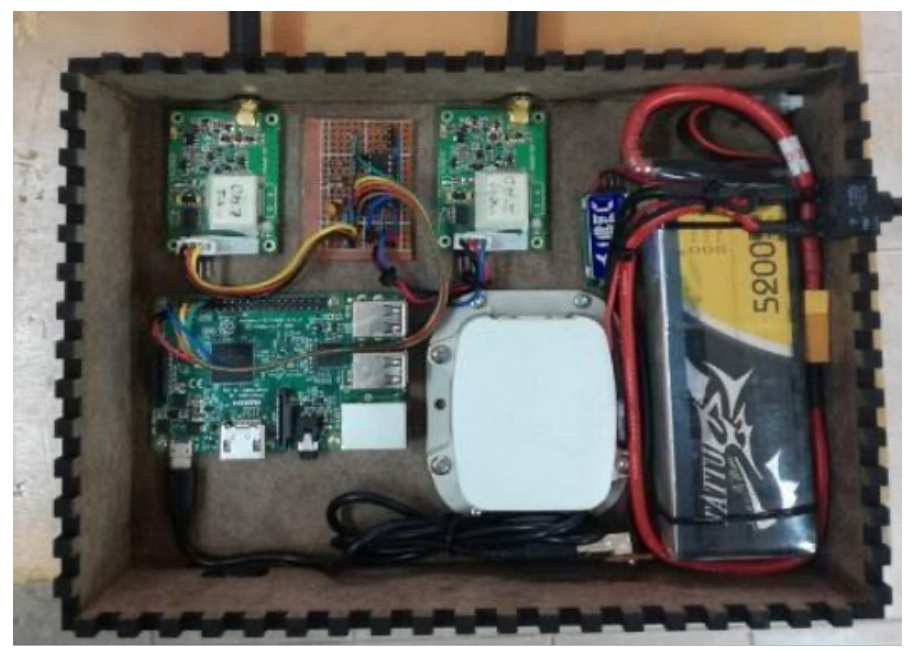

Fuente: elaboración propia 
Pruebas: como protocolo de pruebas del sistema general, se llevó a cabo una serie de mediciones tanto en la parte de interconexión de los subsistemas, teniendo en cuenta cobertura y alcance, como en la capacidad energética para evaluar el desempeño de las baterías en campo de trabajo.

- Cobertura: para esta prueba se delimitó un rango máximo de $3 \mathrm{kms}$ a la redonda del punto seleccionado como estación base, ubicado en el perímetro urbano del municipio de Facatativá, Cundinamarca, Colombia. Y se registró el seguimiento por el reporte del dispositivo satelital SmartOne del nodo receptor en la plataforma Adminsat (Figura 9).

El equipo estableció comunicación por radiofrecuencia y marcación de GPS en la mayoría de los puntos establecidos, solo en un punto no se logró realizar enlace estable, debido a la ubicación geográfica y los obstáculos presentes en el área; además, se determinó que siempre y cuando el nodo remoto no se encuentre en movimiento, el enlace es mucho más eficiente con la estación base y presenta limitaciones con obstáculos demasiado densos, como edificaciones altas.

•FIGURA 9• Mapa satelital del área de prueba

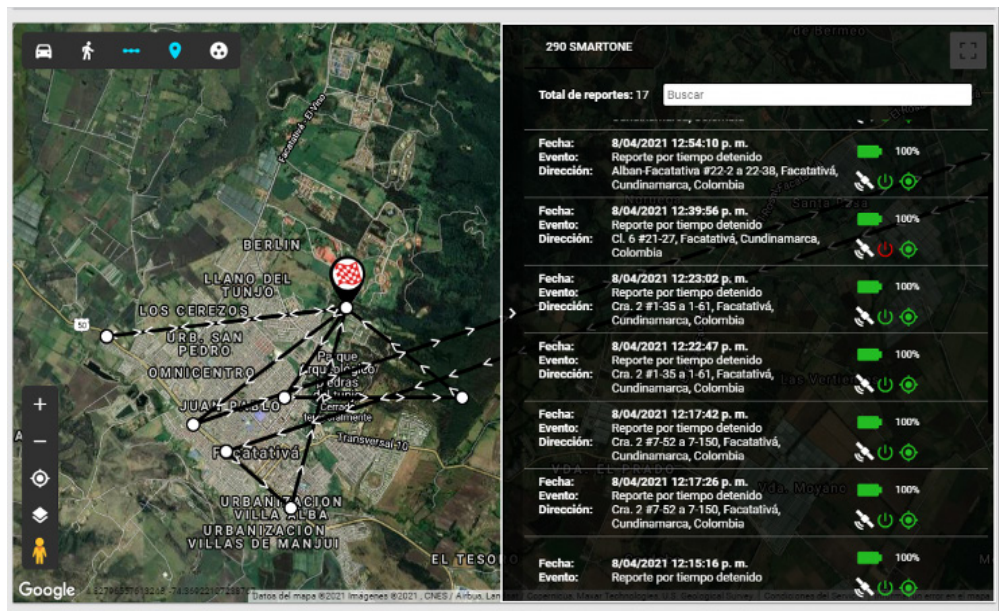

Fuente: registro de seguimiento Plataforma Adminsat

- Alcance: en cuanto a la ejecución de esta prueba, se dispuso como base de operación un cerro ubicado en la vereda Los manzanos, del mismo municipio de Facatativá, en el cual se contaba con línea de vista superior a un rango de $25 \mathrm{kms}$ aproximadamente, medidos desde Google Maps. Allí se ubicó el Nodo Central con sus respectivas antenas directivas para optimizar el enlace, y una persona se desplazó con el Nodo remoto en un vehículo por una ruta seleccionada previamente. 
Como resultado de la prueba se determinó que el equipo logra un enlace eficiente hasta de 10 de $\mathrm{km}$ con los transceiver de $500 \mathrm{~mW}$ y las antenas directivas de 8 $\mathrm{DBi}$; cuando se supera esa distancia, el enlace se hace débil y la comunicación intermitente y defectuosa.

Al igual que en la prueba de cobertura en el entorno urbano, el sistema presenta las limitantes de enlazar la comunicación y reportar a la plataforma satelital cuando hay presencia de obstáculos densos o condiciones climáticas adversas, como lluvia o alta nubosidad.

- Capacidad de Energía: se pone en funcionamiento el sistema completo con solicitud de coordenadas cada $10 \mathrm{~min}$, con el fin de verificar el desempeño de las baterías de alimentación.

Para el Nodo Remoto se usó la batería Marca ULTRA LIFE de litio-Ion a 3.7V, 0.9Ah, 3.4Wh, y se tomaron registros para evaluar el tiempo de descarga (Figura 10).

•FIGURA 10• Gráfica de comportamiento batería Nodo Remoto

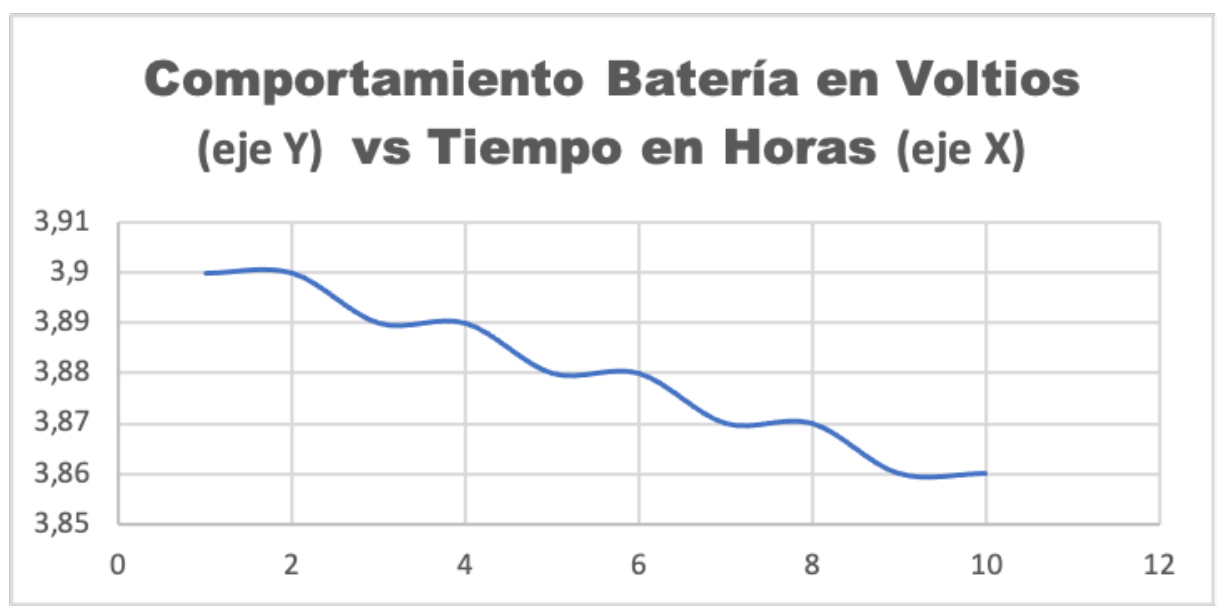

Fuente: elaboración propia.

La batería de Litio-Ion del remoto tuvo un comportamiento de descarga de 5 milivoltios por hora, aproximadamente, bajo las condiciones establecidas.

Para el Nodo Central se usó una batería tipo Polímero de Litio Marca TATUM, 14.8V, 5200mAh, 77Wh. En la figura 11 se observa el comportamiento de descarga de dicha batería. 
-FIGURA 11• Gráfica de comportamiento batería Nodo Central

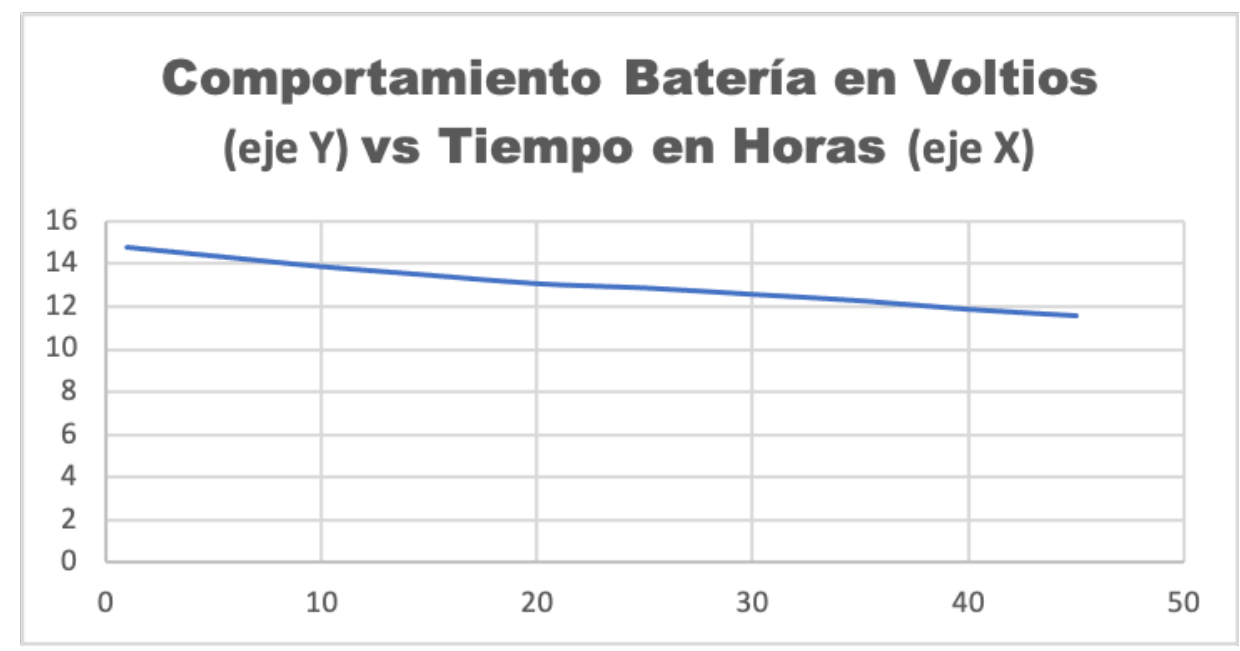

Fuente: elaboración propia.

La batería de Polímero de Litio tuvo un desempeño de trabajo real de 47 horas aproximadamente, bajo las condiciones establecidas, ya que en ese lapso se alcanzó el límite inferior de la batería, lo que hizo que se apagara la unidad central de procesamiento Raspberry PI 3 y, por ende, se detuviera el funcionamiento general del sistema.

\section{Discusión}

La propuesta tecnológica de implementación de los sistemas de geolocalización con módulos de radiofrecuencia es una base fundamental para el desarrollo de aplicaciones en entornos IoT de redes militares, como los detectores de ultrabaja potencia en campos de operación (Fourniol et al., 2018). El estudio de investigación de estos sistemas permite dar una solución a la problemática de uso de equipos de ubicación en zonas donde no existe cobertura de redes de comunicación. Sin embargo, en el desarrollo de pruebas de campo y posibles aplicaciones a nivel operativo se ha podido evidenciar ciertas limitantes, tales como la dificultad para acceder a los puntos donde se pretende instalar el módulo de recepción para garantizar la cobertura del sistema. De igual forma, en el desarrollo de las pruebas se evidenciaron pérdidas en la comunicación entre el Subsistema de Transmisión o Nodo Remoto (NREM) y el Subsistema de Recepción o Nodo Receptor, cuando el nodo remoto se encontraba en desplazamiento.

Así mismo, el sistema está sujeto a pérdidas de comunicación, propios de la propagación dela señal ylas interferencias por otras fuentes de energía electromagnética y obstáculos físicos que atenúan y perturban la señal. Acorde con la investigación se pudieron establecer dos limitaciones en cuanto a la configuración del funcionamiento general, 
por un lado, cuando se programó el sistema con una comunicación unidireccional, en la que el nodo receptor solo recibe los datos del nodo remoto, la frecuencia de marcaciones es mucho más alta y rápida, sin embargo, se aumenta el consumo de energía debido a que no existe una gestión del consumo energético. Por otro lado, cuando se configuró con una comunicación bidireccional en la que el nodo receptor activa y solicita bajo demanda los datos de marcación del nodo remoto, esto permite una mejor administración de energía del nodo remoto, sin embargo, la frecuencia de marcaciones y pérdida de comunicación es mucho más alta.

\section{Conclusiones}

Comercialmente, muchos de los dispositivos dispuestos para la geolocalización de individuos, animales u objetos están limitados en peso y tamaño por el tipo de tecnología que manejan en el envío de información o coordenadas, lo que hace que se dificulte el proceso de implementación para aplicaciones de localización y seguimiento de objetivos; por esta razón las propuestas para dar solución a estas problemáticas se deben dar en una integración completa, que tenga en cuenta varios factores que le puedan dar solución, sin embargo, aunque fue posible proponer un sistema con este concepto de bajas dimensiones e integración de GPS, procesamiento y radiofrecuencia, aún quedan aspectos por mejorar.

Adicionalmente, el manejo o la visualización de la información recopilada por equipos de uso comercial hacen que el tráfico de la misma circule por servidores de operadores o prestadores de servicios de comunicación que no siempre tienen un correcto control o garantía en el manejo de los datos. La implementación del sistema de geolocalización con transmisión de coordenadas vía radiofrecuencia brinda la posibilidad de obtener la información en una central de procesamiento propia en zonas donde las condiciones topográficas limitan el manejo de equipos con tecnología dependiente de redes móviles de comunicación para su normal funcionamiento y que requieren la seguridad y compartimentación del ámbito militar.

La ventaja de contar con un dispositivo programable en el nodo remoto dispuesto en el área de combate es que brinda la posibilidad de interactuar con el equipo para controlar parámetros, como el consumo de batería o los tiempos de marcación y de envío de datos a la central de procesamiento, entre otros, ya que en muchas ocasiones los equipos de geolocalización solo brindan la posibilidad de tener datos cada determinado tiempo por defecto de fábrica.

\section{Referencias}

Bécares, J., Beneharo, R., Arcos, P., \& Ruiz, A. (s.f.). Técnicas de marcaje de aves marinas para el seguimiento remoto. Revista de anillamiento, 25, 26(10), pp.29-40.

Camargo, J., González, L., Segura, D., Garay, F., \& Rincón, N. (2017). Orientación de pasajeros con discapacidad visual dentro del Sistema de Transporte Masivo Transmilenio, mediante Geolocalización Satelital. Ingeniería, 22(2), 283-297. https://doi.org/10.14483/udistrital.jour.reving.2017.2.a08 
Chaves Guerrero, L. M. Jurado Vásquez, H. (2019). E Chaves Guerrero, L. M., \& Jurado Vásquez, H. (2019). El ciberespacio, fuente de control y vigilancia para los ciudadanos. Revista Perspectivas en Inteligencia, 11(20), 347-357. https://doi. org/10.47961/2145194X.391.

Cohen, A. E., Jiang, G. G., Heide, D. A., Pellegrini, V., \& Suri, N. (s.f.). Radio Frequency IoT Sensors in Military Operations in a Smart City.

Cordis. Resultado de la investigación de la UE. (s.f.). Una nueva generación de balizas de socorro con funciones de búsqueda y salvamento basadas en los satélites de Galileo.

Cordis. Comisión Europea. https://cordis.europa.eu/article/id/125182nextgeneration-distress-beacons-will-use-galileo-satellitebased-search-andrescue-capability/es

Corzo, G. D., \& Álvarez-Aros, E. L. (2020). Estrategias de competitividad tecnológica en la conectividad móvil y las comunicaciones de la industria 4.0 en Latinoamérica. Información Tecnológica, 31(6), 183-192. https://doi.org/10.4067/s071807642020000600183

Fourniol, M., Gies, V., Barchasz, V., Kussener, E., \& Glotin, H. (2018). Applications of an Ultra-Low-Power Analog Wake-up Detector for Environmental IoT Networks and Military Smart Dust.

Gazpio, A. M. (06, 06 2018). GNSS y Aumentación pasado, presente y futuro. Estudios de Vigilancia y Prospectiva Tecnológica en el área de Defensa y Seguri. Retrieved 08 06, 2021, from http://www.cefadigital.edu.ar/bitstream/1847939/1609/1/ TEC $1000 \% 202018 \% 20$ GNSS\%20y\% 20Aumentaci\%C3\%B3n \% 20\% 20 Pasado\%20presente\%20y\%20futuro.pdf

Globalstar, Inc. (2015, 02 10). Spot Trace. SPOT. https://www.findmespot.com/es-la/ products-services/spot-trace

Gotarane, V., \& Raskar, S. (2019). IoT Practices in Military Applications.

Jalaian, B., Gregory, T., Niranjan, S., Russell, S., Sadler, L., \& Lee, M. (2018). Evaluating LoRaWAN-based IoT Devices for the Tactical Military Environment.

Olivera, O. F., Cuervo, J. A., \& Giraldo Ramos, F. N. (2011). Sistema de control de posición angular aplicado a dispositivos RF. Visión electrónica, 5(2), 42-58. https://doi.org/10.14483/22484728.3569

Rafael Alejandro, M. S. (2020). Introducción de las tecnologías de localización y los sistemas de geoposicionamiento en el desarrollo de proyectos de internet de las cosas. In Memorias del Programa de Redes-I3CE de calidad, innovación e investigación en docencia universitaria (p. 8). Universitat d'Alacant. Institut de Ciències de l'Educació. https://rua.ua.es/dspace/bitstream/10045/112408/1/MemoriesXarxes-I3CE-2019-20_174.pdf 
Roman, J. et al. Sistema de posicionamiento en interiores utilizando señales de radio estaciones FM comerciales y Deep Learning. Revista Iberoamericana de Automática e Informática industrial, [S.1.], v. 17, n. 1, p. 34-43, ene. 2020. ISSN 1697-7920. Disponible en: <https://polipapers.upv.es/index.php/RIAI/article/view/10894〉.

V. Pellegrini, Fabio Principe, Rodolfo Guidi, Gabriele Scozza, Giacomo de Mauro, \& Riccardo Cioni. (2018). Adding PHY-Layer Crypto to COFDM Radios through a Large Array with Directional Modulation. Globecom., 1(Globecom.), 6.

V. Pellegrini, F. Principe, G. Demauro, R. Guidi, V. Martorelli, \& R. Cioni. (2014, May 4-9). Cryptographically Secure Radios Based on Directional Modulation. IEEE ICASSP, 1 (Florencia, Italia), 6.

Wang, J., Cao, L., Shen, Y., \& Zheng, G. (2018). Research on Design of Military Logistics Support System based on IoT. 